Research Article

\title{
Specific Gene- and MicroRNA-Expression Pattern Contributes to the Epithelial to Mesenchymal Transition in a Rat Model of Experimental Colitis
}

\author{
Éva Boros, ${ }^{1}$ Marianna Csatári, ${ }^{1}$ Csaba Varga, ${ }^{2}$ Balázs Bálint, ${ }^{3}$ and István Nagy ${ }^{1,3}$ \\ ${ }^{1}$ Institute of Biochemistry, Biological Research Centre of the Hungarian Academy of Sciences, Szeged, Hungary \\ ${ }^{2}$ Department of Physiology, Anatomy and Neuroscience, University of Szeged, Szeged, Hungary \\ ${ }^{3}$ Seqomics Biotechnology Ltd., Mórahalom, Hungary \\ Correspondence should be addressed to István Nagy; nagyi@baygen.hu
}

Received 20 January 2017; Accepted 22 March 2017; Published 10 May 2017

Academic Editor: Cecilia Battistelli

Copyright (c) 2017 Éva Boros et al. This is an open access article distributed under the Creative Commons Attribution License, which permits unrestricted use, distribution, and reproduction in any medium, provided the original work is properly cited.

\begin{abstract}
The aim of this study was to determine the gene- and microRNA-expression profile contributing to epithelial to mesenchymal transition in a rat model of experimental colitis. For this, inflammation was induced by injecting 2,4,6trinitrobenzene sulphonic acid to the colon of male Wistar rats. Samples were taken from both inflamed and uninflamed regions of the same colon, total RNA was isolated, and the mRNA and microRNA expressions were monitored. We have determined that the expression of genes responsible for inducing mesenchymal phenotype, such as Egr1, Fgf2, Fgf7, Jak2, Notch2, Hif1 $\alpha$, Zeb2, Mmp9, Lox, and Vim, was all significantly induced in the inflamed regions of the affected colons while the epithelial marker E-cadherin (Cdh1) was downregulated. In contrast, the expression of microRNAs miR-192, miR-143, miR-375, miR-30a, miR-107, and miR-200b responsible for the regulation of the above mentioned genes was significantly downregulated in inflamed colon. Importantly, we detected moderate induction in the expression of five out of six tested microRNAs in the uninflamed regions. In summary, we identified numerous interacting genes and microRNAs with mutually exclusive expression pattern in inflamed regions of colitis-induced rats. These findings suggest that-among others-an important step in the epithelial to mesenchymal transition in experimental colitis is the dysregulated microRNA expression.
\end{abstract}

\section{Introduction}

Inflammatory bowel disease (IBD) is a chronic relapsing disorder of the gastrointestinal tract. The two main clinical appearances of IBD are ulcerative colitis (UC) and Crohn's disease (CD): both cause idiopathic, returning inflammation along the digestive system, where inflamed and uninflamed regions sporadically follow each other. Primary symptoms of this lifelong disease are abdominal pain, diarrhoea, and malabsorption which are significantly affecting the quality of life. Chronic inflammation, the main feature of IBD, is also a well-known factor in cancer progression. In line with this, IBD significantly increases the probability of colorectal cancer $[1,2]$. A crucial step in cancer development and progression of chronic inflammatory diseases is the epithelial to mesenchymal transition (EMT). During EMT cell-cell connections disintegrate, the expression of extracellular matrix components, such as vimentin and metalloproteases, increases and as a consequence cells migrate to the interstitium. Under physiological conditions, EMT is an essential process of wound healing; however, in the absence of proper control, motile cells move through the basal membrane and cause inflammation $[3,4]$. The progression of 


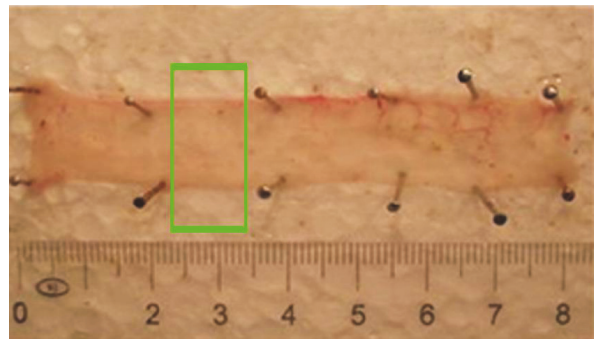

(a)

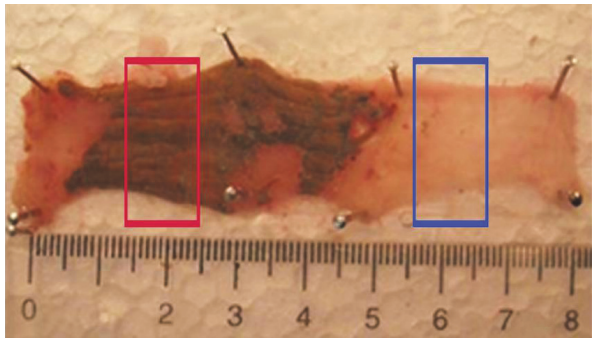

(b)

FiguRE 1: Representative images of noninduced (a) and colitis-induced (b) colons. Green box in (a) indicates control sampling; red and blue boxes in (b) indicate sampling from colitis-induced inflamed and uninflamed regions, respectively.

EMT is regulated by complex molecular mechanisms: transcription factors, such as Snail, Twist, and Zeb, suppress the expression of a well-known epithelial marker E-cadherin (Cdh1). Subsequently, decreased expression of Cdh1 leads to the failure of cell-cell connections; therefore, mesenchymal phenotype emerges. In this process, interrelated signalling pathways, such as Notch and JAK-STAT pathways, also promote the formation of the mesenchymal phenotype [5-9].

Genes known to play role in EMT may be regulated by small, noncoding microRNA (miRNA) molecules, which are directly influencing the translation or degradation of messenger RNAs (mRNAs) by binding to their 3 '-UTRs. miRNAs can also affect epigenetic landscape through the inhibition of DNA methyltransferase genes, thereby causing global gene expression changes [10]. The involvement of miRNAs, such as the miR-200 family members (miR-200a, miR-200b, miR-200c, miR-141, and miR-429), regulating EMT has been shown earlier; in addition, their dysregulated expression was described in several oncologic conditions $[11,12]$. Furthermore, EMT-associated miRNA/mRNA signatures of clear-cell renal cell carcinoma patients highly correlated with clinical stages of cancer progression, which imply the prognostic potential of combined miRNA/ mRNA expression profiling in different EMT-related diseases [13].

Understanding the molecular mechanisms inducing and regulating EMT - such as transcriptional and posttranscriptional modifications - upon chronic intestinal inflammation are critical for understanding the exact pathomechanism of ulcerative colitis and Crohn's disease. In line with this, the aim of this study was to determine the expression patterns of EMT-related genes as well as miRNAs regulating these genes in the inflamed and uninflamed colon regions of the 2,4,6-trinitrobenzene sulphonic acid- (TNBS-) induced rat model of experimental colitis.

\section{Materials and Methods}

2.1. In Vivo Rat Model and Sample Collection. We used male Wistar rats weighing $180-220 \mathrm{~g}$. All rats had access to water and food ad libitum, except overnight before induction of colitis. The animals were randomly divided into two groups: the first group served as control $(n=2$; vehicle-treated hence noncolitis induced) and the second group was induced by 2,4,6-trinitrobenzene sulphonic acid (TNBS) $(n=6$; colitis-induced) based on the method described by Morris et al. [14]. Briefly, TNBS (10 mg in $0.25 \mathrm{ml}$ of $50 \%$ ethanol, $w / v$ ) was intracolonically administered in a volume of $0.25 \mathrm{ml}$, via an $8 \mathrm{~cm}$ long plastic catheter under transient ether anaesthesia. 72 hours after the treatment, all animals were sacrificed and $\sim 8 \mathrm{~cm}$ of distal colons were removed, opened longitudinally, and gently cleaned of fecal content using ice-cold physiological saline. In the case of the control group, since noninflamed, samples were taken from random colon sections (Figure 1(a)). In case of colitis-induced animals, samples were taken from inflamed colon region as well as from nonadjacent uninflamed region (Figure 1(b)). All samples were kept in TRIzol reagent (Thermo Fisher) at $-80^{\circ} \mathrm{C}$.

\subsection{Extraction of Total RNA and Reverse Transcription. Sam-} ples from the colon were homogenized in TRIzol reagent by ULTRA-TURRAX T-18 (IKA) instrument as described previously [15]. $0,1 \mathrm{ml}$ of chloroform (Sigma-Aldrich) was added to $0,3 \mathrm{ml}$ homogenized sample with vigorous vortexing. Samples were centrifuged at $13000 \mathrm{rpm}$ for 10 minutes. Total RNA was than extracted from the upper aqueous phase with RNeasy Plus Mini Kit (Qiagen) according to the manufacturer's protocol. The quality and the quantity of the extracted RNAs were determined by TapeStation (Agilent) and Qubit fluorometer (Thermo Fisher). The extracted total RNA samples were only used if the RNA integrity number (RIN) was greater than 7 .

Reverse transcription was performed by two different kits, depending on the type of RNA to be reverse transcribed. For mRNA detection, cDNA was synthesized using SuperScript VILO Master Mix (Thermo Fisher) according to the manufacturer's instructions. Briefly, at least $70 \mathrm{ng}$ total RNA was combined with 5X VILO Reaction Mix, 10X SuperScript Enzyme Mix, and nuclease-free water. The reaction was incubated at $25^{\circ} \mathrm{C}$ for 10 minutes, then at $42^{\circ} \mathrm{C}$ for 60 minutes, and finally at $85^{\circ} \mathrm{C}$ for 5 minutes. For miRNA detection, TaqMan MicroRNA Reverse Transcription Kit (Thermo Fisher) was used. Briefly, at least $6 \mathrm{ng}$ total RNA per reaction was mixed with the following components: $100 \mathrm{mM}$ dNTPs, MultiScribe Reverse Transcriptase, 10X 
TABLE 1: SybrGreen primer sets used in QPCR experiments.

\begin{tabular}{lcc}
\hline Gene & Forward $\left(5^{\prime}-3^{\prime}\right)$ & Reverse $\left(5^{\prime}-3^{\prime}\right)$ \\
\hline Egr1 & AACAACCCTACGAGCACCTG & AAAGGGGTTCAGGCCACAAA \\
Fgf2 & GCGACCCACACGTCAAACTA & CCGTGACCGGTAAGTGTTGTA \\
Fgf7 & TGTGGCAATCAAAGGGGTGG & AAGGCCACGAACATTTCCCC \\
Jak2 & AGTGTGCTACAGTGCTGGTC & TTCCTTGTTGCCAGATCCCG \\
Mmp9 & GCCGGGAACGTATCTGGAAA & GGTTGTGGAAACTCACACGC \\
Notch2 & AACTGCACCTCCTCACTTCG & CTCCTCGTTGTTGCATCCCT \\
Vim & CATGCGGCTGCGAGAAAAAT & GGTCAAGACGTGCCAGAGAA \\
Zeb2 & AAAGCAGTTCCCTTCTGCGA & AGGAGCCCGAGTGTGAAAAG \\
Hif1 $\alpha$ & CTCATCCAAGGAGCCTTAACCT & TAACGTTCCAATTCCTGCTGC \\
Cdh 1 & AGGGCGGATGTCAGAGACTA & CATCCAGCAGGTCGTAGTGG \\
\hline
\end{tabular}

Reverse Transcription Buffer, RNase inhibitor, and nucleasefree water. Revers transcription reactions were performed under the following PCR conditions: 30 minutes at $16^{\circ} \mathrm{C}, 30$ minutes at $42^{\circ} \mathrm{C}$, and 5 minutes at $85^{\circ} \mathrm{C}$.

2.3. Quantitative Real-Time PCR (QRT-PCR). miRNA or mRNA expression was measured by real-time PCR using the StepOne PCR Systems (Thermo Fisher). SybrGreen and TaqMan technology-based quantitative real-time PCR was used to quantify the relative abundance of the selected mRNAs. For this, specific exon spanning primer sets were used as listed in Table 1. As controls, we used reaction mixtures without cDNA. Briefly, amplification was carried out in a total volume of $6 \mu \mathrm{l}$, containing $1 \mu \mathrm{l}$ of $\mathrm{cDNA}, 3 \mu \mathrm{l}$ of SYBR Select Master Mix $(2 \times), 1,04 \mu \mathrm{l}$ nuclease-free water, $0,48 \mu \mathrm{l}$ of forward, and $0,48 \mu \mathrm{l}$ reverse primer. The QRTPCR reactions were performed under the following PCR conditions: one cycle of 2 minutes at $50^{\circ} \mathrm{C}$ and 2 minutes at $95^{\circ} \mathrm{C}$, followed by 40 cycles of $15 \mathrm{sec}$ each at $95^{\circ} \mathrm{C}$, and 1 minute at $60^{\circ} \mathrm{C}$. Detection of nonspecific amplification was carried out by melt curve analysis. TaqMan technology-based reactions were performed in total volume of $4 \mu \mathrm{l}$, containing $1 \mu \mathrm{l}$ cDNA, $2 \mu \mathrm{l}$ of TaqMan Fast Advanced Master Mix (2×), 0,2 $\mu$ l TaqMan Gene Expression Assay, and 0,8 $\mu \mathrm{l}$ nuclease-free water. Thermal-cycling conditions were as follows: one cycle of 2 minutes at $50^{\circ} \mathrm{C}$ and $20 \mathrm{sec}$ at $95^{\circ} \mathrm{C}$ and followed by 40 cycles of $1 \mathrm{sec}$ at $95^{\circ} \mathrm{C}$ and $20 \mathrm{sec}$ at $60^{\circ} \mathrm{C}$. All of the measurements were performed in duplicate with at least two biological replicates. The ratio of each mRNA relative to the $18 \mathrm{~S}$ rRNA (Thermo Fisher assay number hs-99999901_s1) was calculated using the $2^{-\Delta \Delta \mathrm{CT}}$ method.

For measurement of miRNA expression levels, TaqMan Universal PCR Master Mix (Thermo Fisher) was used according to the manufacturer's instructions. Briefly, amplification was carried out in a total volume of $15 \mu \mathrm{l}$ containing the following components: $1 \mu \mathrm{l}$ miRNA specific cDNA, 0,75 $\mu$ l TaqMan Small RNA Assay $(20 \times), 7,52 \mu \mathrm{l}$ TaqMan Universal PCR Master Mix (2×), and 5,73 $\mu \mathrm{l}$ nuclease-free water. Thermal-cycling conditions were as follows: one cycle of 10 minutes at $95^{\circ} \mathrm{C}, 40$ cycles of
TABLE 2: miRNA specific TaqMan microRNA assays.

\begin{tabular}{lc}
\hline miRNA & Assay number \\
\hline miR-125a & 002198 \\
miR-192 & 000491 \\
miR-200b & 002251 \\
miR-375 & 000564 \\
miR-30a & 000417 \\
miR-107 & 000443 \\
miR-143 & 463509 \\
U6 snRNA & 001973 \\
\hline
\end{tabular}

$15 \mathrm{sec}$ at $95^{\circ} \mathrm{C}$, and 1 minute at $60^{\circ} \mathrm{C}$. The specific miRNA assays were purchased from Thermo Scientific; assay numbers are shown in Table 2. The ratio of each miRNA relative to the endogenous U6 snRNA was calculated using the $2^{-\Delta \Delta C T}$ method.

2.4. Statistical Analysis and Data Representation. Statistical evaluations were performed using the IBM SPSS Statistics program for Windows. Graphs were plotted with GraphPad Prism 6 software. Quantitative data are presented as the mean \pm SEM, and the significance of difference between sets of data was determined by one-way analysis of variance (ANOVA) following LSD post hoc test; a $p$ value of less than 0.05 was considered significant.

\section{Results and Discussion}

In order to investigate whether EMT is involved in the pathogenesis of chronic colon inflammation, we used the 2,4,6-trinitrobenzene sulfonic acid- (TNBS-) induced rat model of colitis, in which the inflammatory response is due to the generation of transmural oxidative stress and release of proinflammatory mediators [16, 17]. We hypothesized that even though only marked regions of the colon show severe inflammation (Figure 1(b)), 


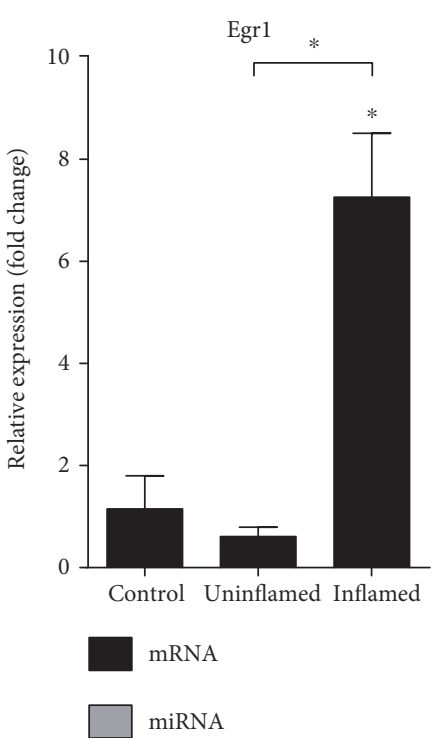

(a)

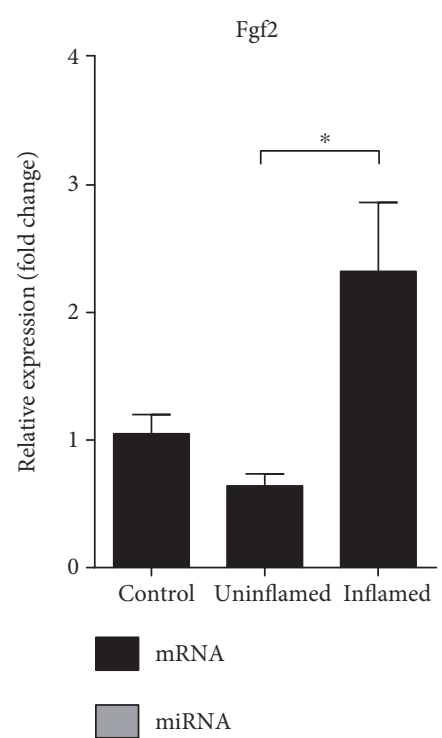

(b)

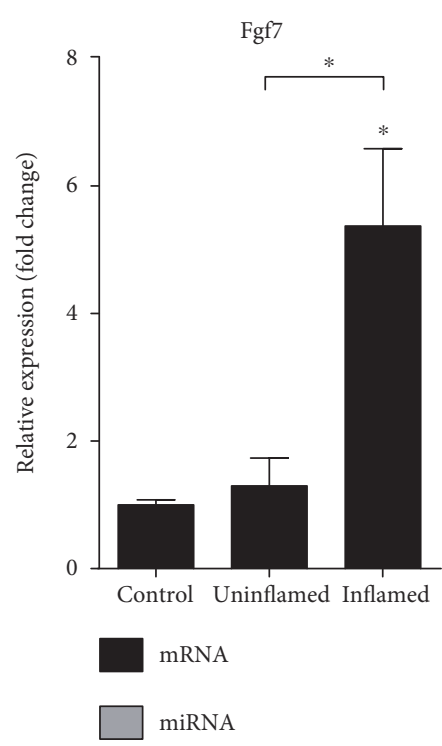

(c)

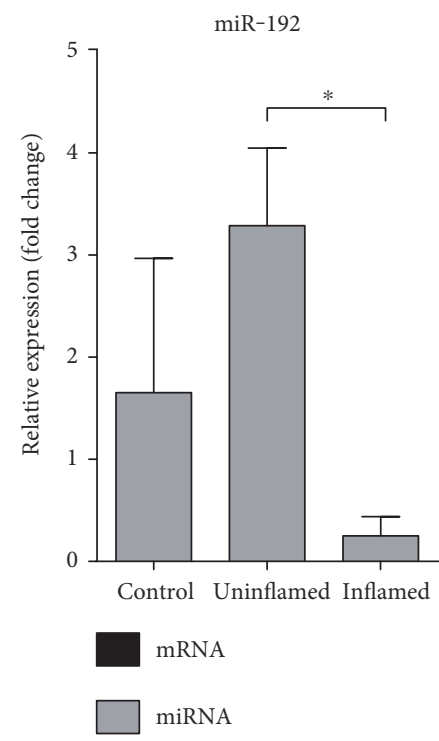

(d)

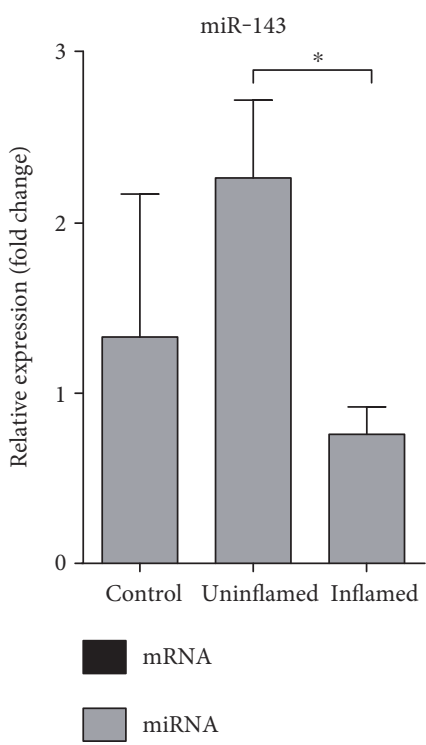

(e)

FIGURE 2: Opposing expression of growth factors and their regulating miRNAs at the site of colon inflammation. The relative gene expression of growth factors Egr1 (a), Fgf2 (b), and Fgf7 (c) shows significant upregulation in inflamed colons as compared to both control and uninflamed colons of colitis-induced animals. In contrast, the expression pattern of both miR-192 (d) and miR-143 (e) shows slight upregulation in uninflamed colons and a significant downregulation in inflamed colons. Data are presented as the mean \pm SEM; the significance of differences between sets of data was determined by one-way analysis of variance (ANOVA) using SPSS Statistics; ${ }^{*} p<0.05$.

proximal, phenotypically not inflamed regions may also be affected. Hence, we decided to take two samples from the colons of the colitis-induced animals: one sample corresponds to the severely inflamed region and the other to noninflamed region, referred as inflamed and uninflamed, respectively; the two regions are being separated by at least $2 \mathrm{~cm}$ from each other (Figure 1(b)). Subsequently, we determined the expression profile of selected genes and microRNAs related to EMT.

3.1. Induced Expression of Growth Factors at the Site of Induced Colitis. Signalling pathways activating the formation of motile mesenchymal cells are induced by growth factors, such as the early growth response protein 1 (Egr1) and fibroblast growth factors 2 and 7 (Fgf2, Fgf7) $[18,19]$. All three genes showed increased expression in inflamed region of the colitis-induced colons as compared to both controls and noninflamed regions (Figures 2(a)$2(c))$. It is known that miR-192 regulates the expression of Egr1 and Fgf2, while miR-143 is a known inhibitor of Fgf7 [20]. As compared to controls, both miRNAs are slightly but not significantly induced in uninflamed regions and supressed in inflamed regions. When comparing the expression in uninflamed versus inflamed regions, 


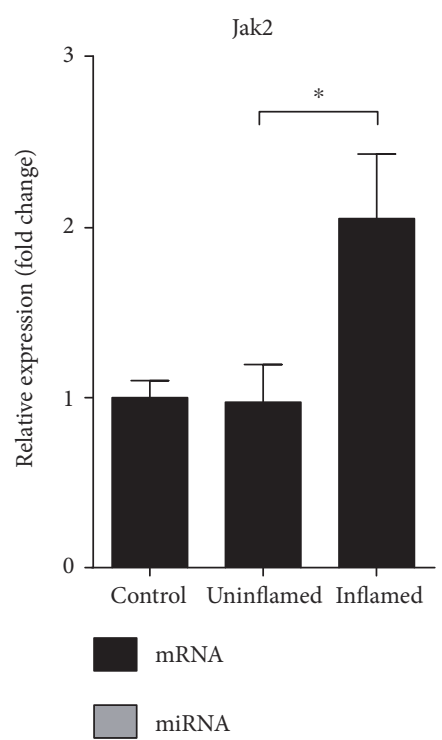

(a)

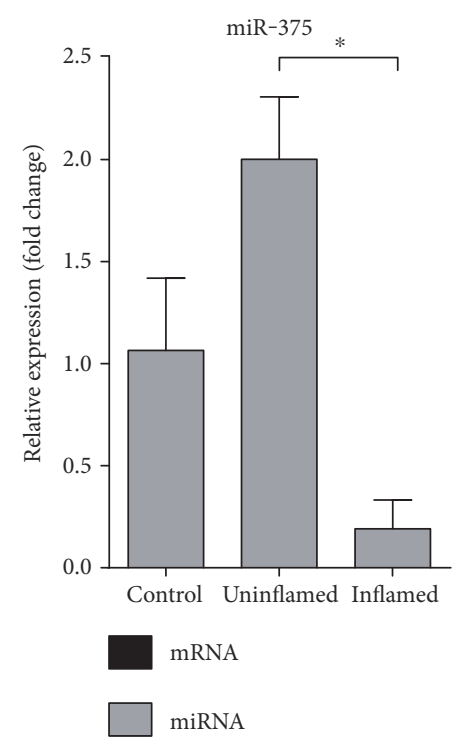

(d)

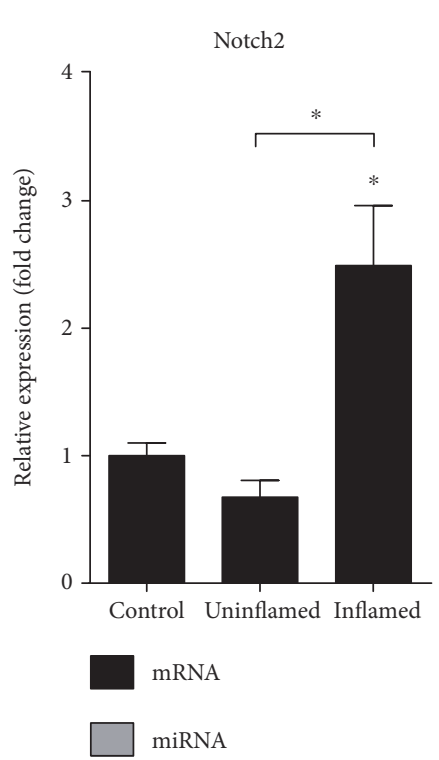

(b)

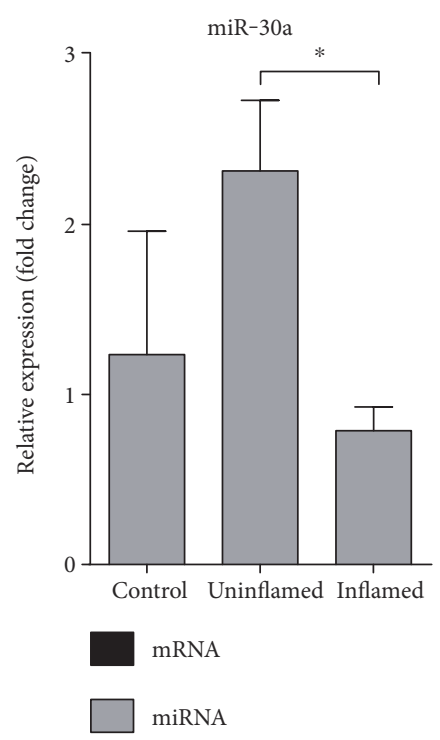

(e)

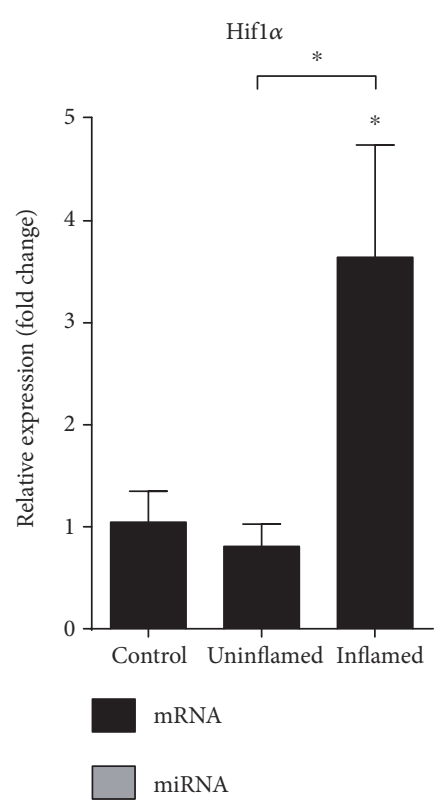

(c)

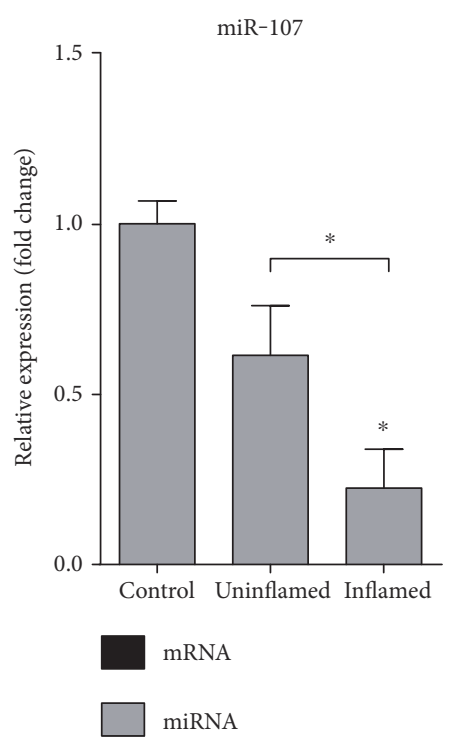

(f)

FIGURE 3: Induced expression of genes regulating proliferation occurs due to decreased expression of miRNAs. The relative gene expression of Jak2 (a), Notch2 (b), and Hif1 $\alpha$ (c) shows significant upregulation in inflamed colons as compared to both control and uninflamed colons of colitis-induced animals. In contrast, the expression of miR-375 (d), miR-30a (e), and miR-107 (f) is significantly downregulated in inflamed colons. Data are presented as the mean \pm SEM; the significance of differences between sets of data was determined by one-way analysis of variance (ANOVA) using SPSS Statistics; ${ }^{*} p<0.05$.

we detected significant suppression in inflamed regions (Figures 2(d) and 2(e)). The negative correlation between the gene and microRNA expression suggests that the increased mRNA levels of growth factors (Egr1, Fgf2, and Fgf7) may be due to the downregulated expression of their regulating microRNAs (miR-192, miR-143).

3.2. JAK/STAT and Notch Signalling Pathways Are Activated upon Inflammation of the Colon. We next monitored the expression profile of key molecules belonging to the JAK/STAT and Notch pathways that play important roles in the regulation of expression of transcription factors associated with EMT $[6,9]$. We observed that both Janus kinase 2 (Jak2) and Notch2 were upregulated in the colitis-induced inflamed colon regions (Figures 3(a) and 3(b)). Ding et al. earlier demonstrated that miR-375 directly targets the Jak2 oncogene, thereby regulating cancer cell proliferation [21]. In our model, the amount of miR-375 was significantly decreased in the inflamed region, while elevated in the TNBS treated uninflamed sections (Figure 3(d)). When monitoring the expression of miR-30a, the endogenous inhibitor of Notch2 expression 


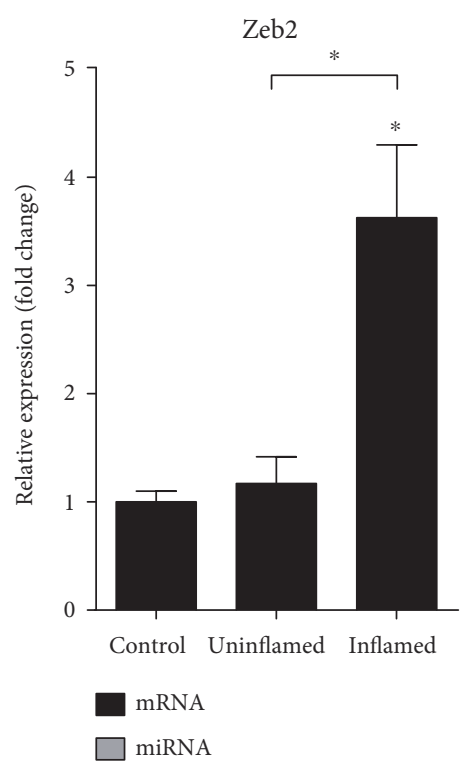

(a)

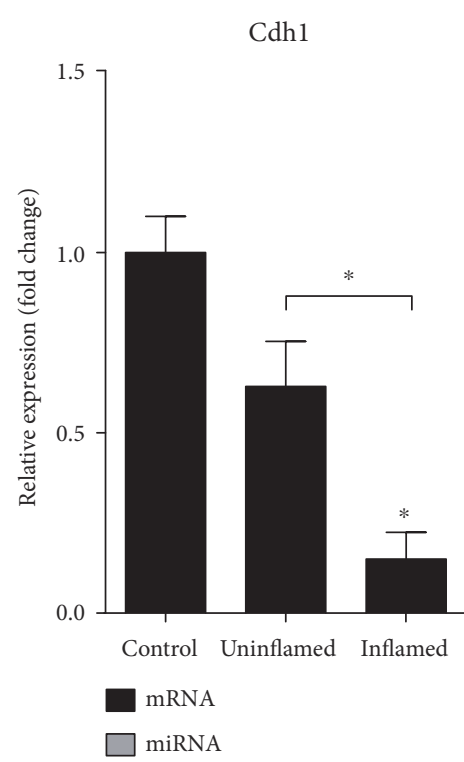

(b)

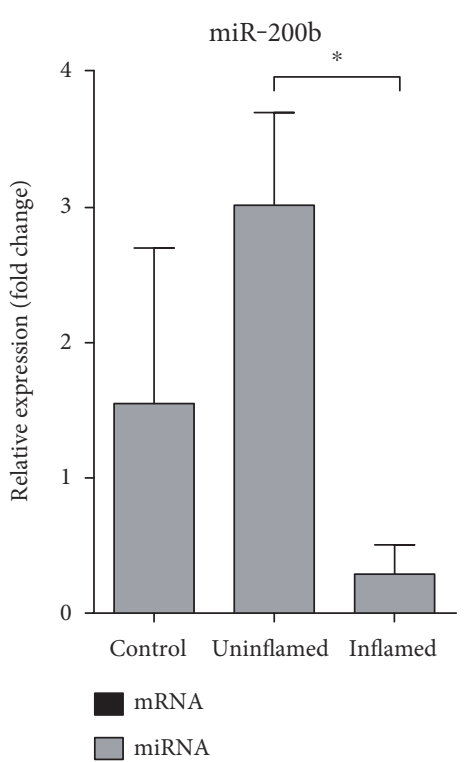

(c)

Figure 4: Induced expression of Zeb2 in inflamed colon downregulates the expression of its targets Cdh1 and miR-200b. The relative gene expression of Zeb2 (a) is significantly induced in inflamed colons as compared to both control and uninflamed colons of colitis-induced animals. In contrast, epithelial markers Cdh1 (b) and miR-200b (c) are both significantly downregulated in inflamed colons of colitis-induced animals. Data are presented as the mean \pm SEM; the significance of differences between sets of data was determined by one-way analysis of variance (ANOVA) using SPSS Statistics; ${ }^{*} p<0.05$.

[22], we again detected significant decrease in inflamed samples (Figure 3(e)).

Notch pathway is also induced by hypoxia; therefore, we next examined the expression of a well-known marker of hypoxia and oxidative stress, hypoxia induced factor $1 \alpha$ (Hifl $\alpha)$ [6]. mRNA level of Hifl $\alpha$ was again significantly induced in inflamed region of the colon (Figure 3(c)). In contrast, the expression of miR-107, a common regulator of Notch2 and Hif1 $\alpha[23,24]$, decreased in colitis-induced samples (Figure 3(f)). These data suggest that the absence of inhibition caused by decreased miRNA expression in inflamed regions may shift cell proliferation balancing pathways towards EMT.

3.3. Cells Lose Epithelial Nature at the Site of Colon Inflammation. Transcription factors involved in the progression of EMT are, among others, Snail, Twist, and Zeb [25]. Zeb2 has a fundamental role in the expressional inhibition of E-cadherin coding gene, Cdh1 [26]. This process is under the control of a well described regulatory feedback loop between Zeb2 and miR-200b; importantly, Zeb2 and miR-200b can also inhibit each other [27]. To prove if EMT was indeed induced after colitis induction, we first determined the expression of epithelial marker Cdh1 and its regulator Zeb2. While the mRNA level of Zeb2 was markedly upregulated at the site of inflammation, Cdh1 was significantly downregulated (Figures 4(a) and 4(b)). The expression of miR-200b also inversely correlated with the expression of Zeb2, being markedly downregulated in inflamed regions (Figure 4(c)). It is important to note that miR-192, also known to regulate the expression of Zeb2 [28], exhibits similar expression profile as miR-200b (Figure 2(d)). These data show that the expression of Zeb2 is de-repressed at the site of colonic inflammation due to the decreased expression of its microRNA regulators $\mathrm{miR}-200 \mathrm{~b}$ and $\mathrm{miR}-192$. Increased Zeb2 expression, in turn, downregulates epithelial marker Cdh1 expression, and as a result, cells lose their connections as well as apical-basal polarity which all act in favour of epithelial to mesenchymal transition.

3.4. Colon Inflammation Is Marked by Gain of Mesenchymal Characteristics. A well-known marker of EMT is the expression of the mesenchymal marker vimentin (Vim) [29]. Furthermore, a prerequisite of cell invasion is the rearrangement of microenvironment by extracellular enzymes, such as matrix metalloproteinase 9 (Mmp9) and lysyl oxidase (Lox) [8]. We have determined that Mmp9, Lox, and Vim all show significantly induced expression in inflamed colon (Figures 5(a)-5(c)) pointing toward gain of mesenchymal phenotype at the site of colon inflammation. Again, miR125a, miR-30a, and miR-200b regulating Mmp9, Lox, and Vim, respectively [30-32], are all downregulated in inflamed region as compared to uninflamed colon (Figures 3(e), 4(c), and $5(\mathrm{~d})$ ). Altogether, these expressional changes confirm that epithelial to mesenchymal transition indeed takes place in colitis induced rats.

\section{Conclusion}

Our present study demonstrates that the epithelial to mesenchymal transition is activated during colitis induction in rats. 

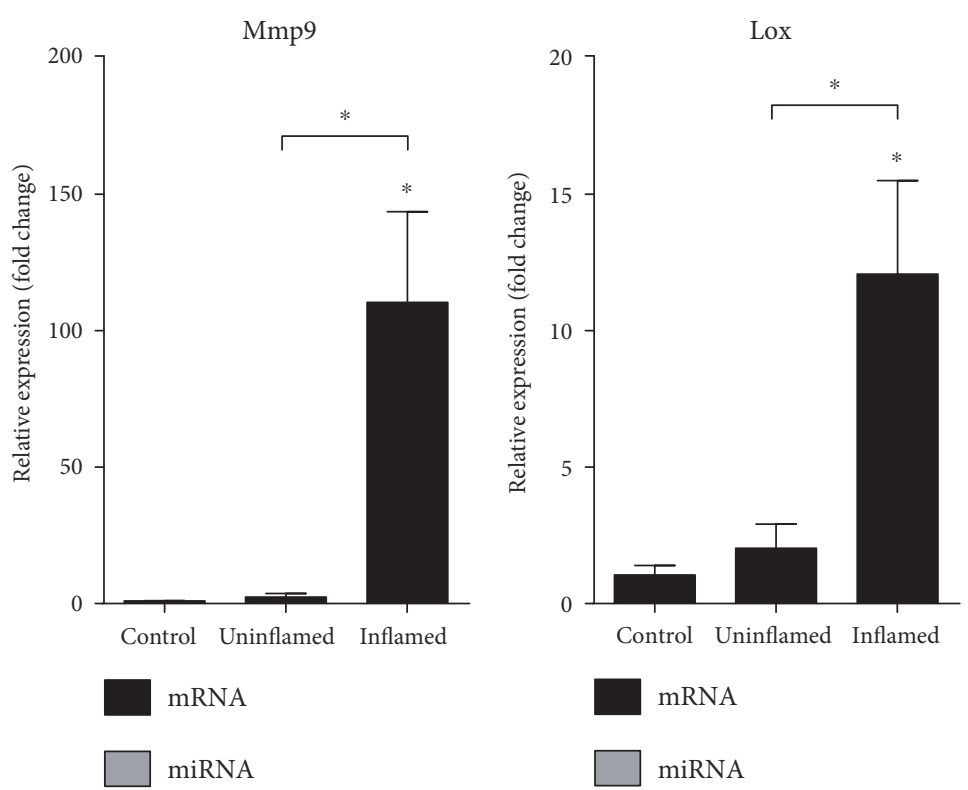

(a)

(b)
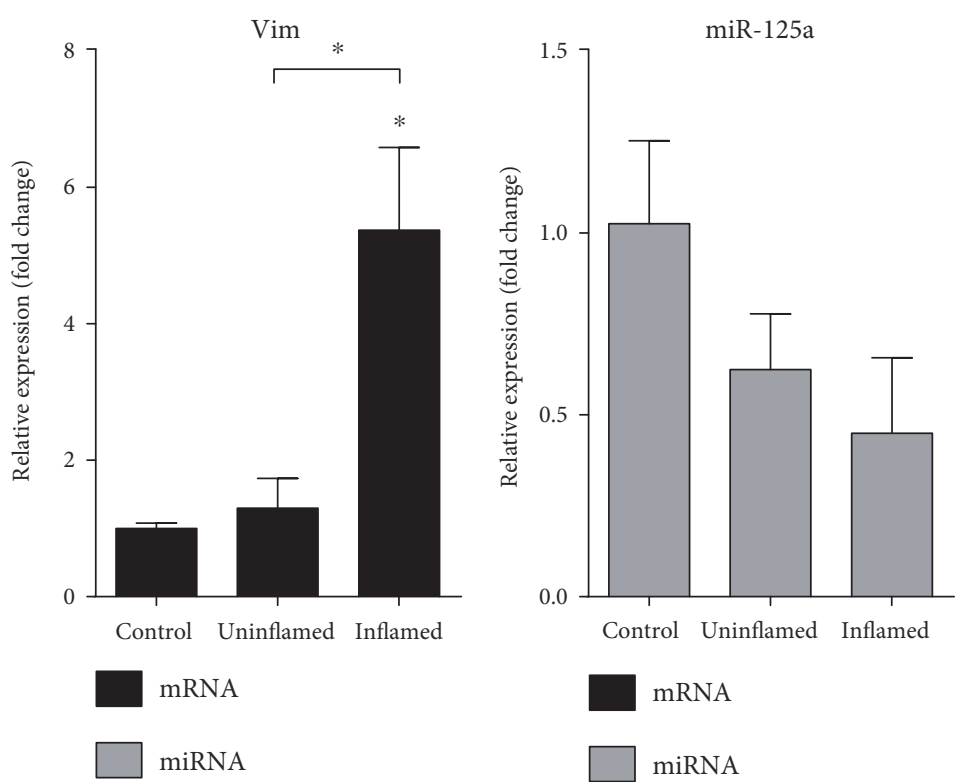

(c)

(d)

FIGURE 5: The expression of mesenchymal markers Mmp9, Lox, and Vim is upregulated at the site of colon inflammation. The relative gene expression of Mmp9 (a), Lox (b), and Vim (c) is significantly induced in inflamed colons as compared to both control and uninflamed colons of colitis-induced animals. Conversely, mir-125a (d) shows slight but not significant downregulation in both uninflamed and inflamed colons $(p=0.285$ and $p=0.135$, resp.). Data are presented as the mean \pm SEM; the significance of differences between sets of data was determined by one-way analysis of variance (ANOVA) using SPSS Statistics; ${ }^{*} p<0.05$.

The presented data indicate that, among others, the activation takes place due to the opposed expression profile of genes and their regulating microRNAs at the site of inflammation (Figure 6); while the expression of all tested mesenchymal markers (Egr1, Fgf2, Fgf7, Jak2, Notch2, Hif1 $\alpha$, Zeb2, Mmp9, Lox, and Vim) was significantly induced, microRNAs regulating their expression decreased (miR-192, miR-143, miR-375, miR-30a, miR-107, miR200b, and miR-125a). In parallel, the expression of E-cadherin, a specific marker of epithelial cells, also decreased further supporting the loss of epithelial phenotype at the site of inflammation. These findings shed light onto a microRNA-mediated regulation influencing epithelial to mesenchymal transition in experimental colitis.

\section{Perspectives}

The number of miRNAs shown to be downregulated in IBD is increasing. Elevating the level of these cellular miRNAs using miRNA mimics holds the potential to become a true 


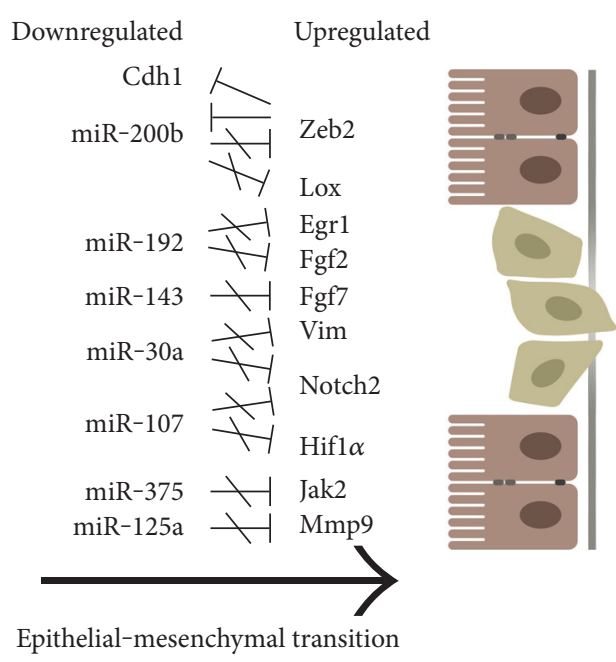

FIgURE 6: Characteristic features of epithelial to mesenchymal transition at the site of colon inflammation in TNBS-induced rat model of experimental colitis. Because of the downregulated expression, microRNAs are no longer able to regulate the expression of their target genes at the site of colonic inflammation; hence, the expression of genes specific for mesenchymal phenotype increases. Upregulated Zeb2 expression, in turn, downregulates the expression of Cdh1 which further promotes the loss of epithelial phenotype.

alternative for therapeutic restoration of physiological pathways lost in IBD. Since miRNAs target multiple genes, restoration of downregulated miRNAs has the potential to have a greater therapeutic effect than drugs with a single protein target [33]. Indeed, recent successful phase IIa studies of miRNA therapy in humans are all in favour of miRNAs being potential therapeutic targets in IBD.

Emerging data also supports the involvement of another class of noncoding RNAs, the long noncoding RNAs, in the epithelial-to-mesenchymal transition. It has recently been shown that HOTAIR (HOX Transcript Antisense Intergenic RNA) mediates a physical interaction between Snail and EZH2 (Enhancer of zeste homolog 2) and thereby influences EMT [34]. In addition, multiple lncRNAs are differentially expressed in IBD and are regulating cellular physiology [35]. Whether lncRNAs may be potential therapeutic targets in IBD remains to be determined.

\section{Conflicts of Interest}

The authors have no competing interests to declare.

\section{Authors' Contributions}

Éva Boros and István Nagy conceived and supervised the project and designed the experiments. Csaba Varga performed experiments with rats. Éva Boros and Marianna Csatári performed all other experiments. Éva Boros, Marianna Csatári, Balázs Bálint, and István Nagy analysed and interpreted data. Éva Boros, Marianna Csatári, and István Nagy wrote the paper.

\section{Acknowledgments}

The authors thank Dr. Zita Szalai for the help with the animal experiments. This work was funded, in part, by grant from the National Research, Development and Innovation Office (Grant no. GINOP-2.3.2-15-2016-00039). Éva Boros was funded by the European Union and the State of Hungary, cofinanced by the European Social Fund in the framework of "National Excellence Program" (Grant no. A2-ELMH12-0082); István Nagy was supported by the János Bolyai Research Scholarship of the Hungarian Academy of Sciences.

\section{References}

[1] B. Khor, A. Gardet, and R. J. Xavier, "Genetics and pathogenesis of inflammatory bowel disease," Nature, vol. 474, no. 7351, pp. 307-317, 2011.

[2] A. Kaser, S. Zeissig, and R. S. Blumberg, "Inflammatory bowel disease," Annual Review of Immunology, vol. 28, no. 1, pp. 573-621, 2010.

[3] G. Y. Yang, S. Taboada, and J. Liao, "Inflammatory bowel disease: a model of chronic inflammation-induced cancer," Methods in Molecular Biology, vol. 511, pp. 193-233, 2009.

[4] M. Zeisberg and E. G. Neilson, "Biomarkers for epithelialmesenchymal transitions," The Journal of Clinical Investigation, vol. 119, no. 6, pp. 1429-1437, 2009.

[5] A. Cano, M. A. Perez-Moreno, I. Rodrigo et al., "The transcription factor snail controls epithelial-mesenchymal transitions by repressing E-cadherin expression," Nature Cell Biology, vol. 2, no. 2, pp. 76-83, 2000.

[6] Z. Wang, Y. Li, D. Kong, and F. H. Sarkar, "The role of Notch signaling pathway in epithelial-mesenchymal transition (EMT) during development and tumor aggressiveness," Current Drug Targets, vol. 11, no. 6, pp. 745-751, 2010.

[7] J. Comijn, G. Berx, P. Vermassen et al., "The two-handed E box binding zinc finger protein SIP1 downregulates Ecadherin and induces invasion," Molecular Cell, vol. 7, no. 6, pp. 1267-1278, 2001.

[8] S. Lamouille, J. Xu, and R. Derynck, "Molecular mechanisms of epithelial-mesenchymal transition," Nature Reviews Molecular Cell Biology, vol. 15, no. 3, pp. 178-196, 2014.

[9] A. Yadav, B. Kumar, J. Datta, T. N. Teknos, and P. Kumar, "IL6 promotes head and neck tumor metastasis by inducing epithelial-mesenchymal transition via the JAK-STAT3SNAIL signaling pathway," Molecular Cancer Research, vol. 9, no. 12, pp. 1658-1667, 2011.

[10] C. Cicchini, V. de Nonno, C. Battistelli et al., "Epigenetic control of EMT/MET dynamics: HNF4alpha impacts DNMT3s through miRs-29," Biochimica et Biophysica Acta, vol. 1849, no. 8, pp. 919-929, 2015.

[11] A. Zaravinos, "The regulatory role of microRNAs in EMT and cancer," Journal of Oncology, vol. 2015, Article ID 865816, p. 13, 2015.

[12] E. C. Lai, "Micro RNAs are complementary to 3 'UTR sequence motifs that mediate negative post-transcriptional regulation," Nature Genetics, vol. 30, no. 4, pp. 363-364, 2002.

[13] H. Mlcochova, T. Machackova, A. Rabien et al., "Epithelialmesenchymal transition-associated microRNA/mRNA signature is linked to metastasis and prognosis in clear-cell renal cell carcinoma," Scientific Reports, vol. 6, p. 31852, 2016. 
[14] G. P. Morris, P. L. Beck, M. S. Herridge, W. T. Depew, M. R. Szewczuk, and J. L. Wallace, "Hapten-induced model of chronic inflammation and ulceration in the rat colon," Gastroenterology, vol. 96, no. 3, pp. 795-803, 1989.

[15] Z. Szalai, A. Szasz, I. Nagy et al., “Anti-inflammatory effect of recreational exercise in TNBS-induced colitis in rats: role of NOS/HO/MPO system," Oxidative Medicine and Cellular Longevity, vol. 2014, Article ID 925981, p. 11, 2014.

[16] X. Z. Shi, J. H. Winston, and S. K. Sarna, "Differential immune and genetic responses in rat models of Crohn's colitis and ulcerative colitis," American Journal of Physiology Gastrointestinal and Liver Physiology, vol. 300, no. 1, pp. G41-G51, 2011.

[17] R. J. Xavier and D. K. Podolsky, "Unravelling the pathogenesis of inflammatory bowel disease," Nature, vol. 448, no. 7152, pp. 427-434, 2007.

[18] J. Chen, G. Chen, Z. Yan et al., "TGF-beta1 and FGF2 stimulate the epithelial-mesenchymal transition of HERS cells through a MEK-dependent mechanism," Journal of Cellular Physiology, vol. 229, no. 11, pp. 1647-1659, 2014.

[19] M. Kinehara, S. Kawamura, S. Mimura et al., "Protein kinase C-induced early growth response protein-1 binding to SNAIL promoter in epithelial-mesenchymal transition of human embryonic stem cells," Stem Cells and Development, vol. 23, no. 18, pp. 2180-2189, 2014.

[20] S. Y. Wu, R. Rupaimoole, F. Shen et al., "A miR-192-EGR1HOXB9 regulatory network controls the angiogenic switch in cancer," Nature Communications, vol. 7, p. 11169, 2016.

[21] L. Ding, Y. Xu, W. Zhang et al., "MiR-375 frequently downregulated in gastric cancer inhibits cell proliferation by targeting JAK2," Cell Research, vol. 20, no. 7, pp. 784-793, 2010.

[22] M. Ortega, H. Bhatnagar, A. P. Lin et al., "A microRNAmediated regulatory loop modulates NOTCH and MYC oncogenic signals in B- and T-cell malignancies," Leukemia, vol. 29, no. 4, pp. 968-976, 2015.

[23] Y. F. Huang, C. H. Yang, C. C. Huang, and K. S. Hsu, "Vascular endothelial growth factor-dependent spinogenesis underlies antidepressant-like effects of enriched environment," The Journal of Biological Chemistry, vol. 287, no. 49, pp. 4093840955, 2012.

[24] L. Chen, R. Zhang, P. Li et al., "P53-induced microRNA-107 inhibits proliferation of glioma cells and down-regulates the expression of CDK6 and Notch-2," Neuroscience Letters, vol. 534, pp. 327-332, 2013.

[25] B. De Craene and G. Berx, "Regulatory networks defining EMT during cancer initiation and progression," Nature Reviews. Cancer, vol. 13, no. 2, pp. 97-110, 2013.

[26] T. S. Wong, W. Gao, and J. Y. Chan, "Transcription regulation of E-cadherin by zinc finger E-box binding homeobox proteins in solid tumors," BioMed Research International, vol. 2014, Article ID 921564, p. 10, 2014.

[27] C. P. Bracken, P. A. Gregory, N. Kolesnikoff et al., “A doublenegative feedback loop between ZEB1-SIP1 and the microRNA-200 family regulates epithelial-mesenchymal transition," Cancer Research, vol. 68, no. 19, pp. 7846-7854, 2008.

[28] T. Kim, A. Veronese, F. Pichiorri et al., "p53 regulates epithelial-mesenchymal transition through microRNAs targeting ZEB1 and ZEB2," The Journal of Experimental Medicine, vol. 208, no. 5, pp. 875-883, 2011.

[29] C. Y. Liu, H. H. Lin, M. J. Tang, and Y. K. Wang, "Vimentin contributes to epithelial-mesenchymal transition cancer cell mechanics by mediating cytoskeletal organization and focal adhesion maturation," Oncotarget, vol. 6, no. 18, pp. 15966-15983, 2015.

[30] Z. Fan, H. Cui, X. Xu et al., "MiR-125a suppresses tumor growth, invasion and metastasis in cervical cancer by targeting STAT3," Oncotarget, vol. 6, no. 28, pp. 25266-25280, 2015.

[31] M. Sun, S. Gomes, P. Chen et al., "RKIP and HMGA2 regulate breast tumor survival and metastasis through lysyl oxidase and syndecan-2," Oncogene, vol. 33, no. 27, pp. 3528-3537, 2014.

[32] Z. Liu, L. Chen, X. Zhang et al., "RUNX3 regulates vimentin expression via miR-30a during epithelial-mesenchymal transition in gastric cancer cells," Journal of Cellular and Molecular Medicine, vol. 18, no. 4, pp. 610-623, 2014.

[33] C. G. Chapman and J. Pekow, "The emerging role of miRNAs in inflammatory bowel disease: a review," Therapeutic Advances in Gastroenterology, vol. 8, no. 1, pp. 4-22, 2015.

[34] C. Battistelli, C. Cicchini, L. Santangelo et al., "The Snail repressor recruits $\mathrm{EZH} 2$ to specific genomic sites through the enrollment of the lncRNA HOTAIR in epithelial-tomesenchymal transition," Oncogene, vol. 36, no. 7, pp. 942-955, 2017.

[35] F. Wu, Y. Huang, F. Dong, and J. H. Kwon, "Ulcerative colitis-associated long noncoding RNA, BC012900, regulates intestinal epithelial cell apoptosis," Inflammatory Bowel Diseases, vol. 22, no. 4, pp. 782-795, 2016. 


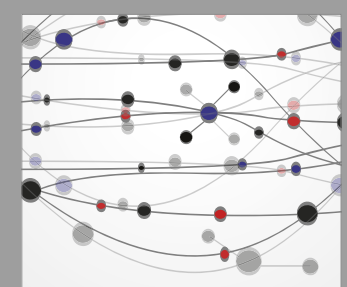

The Scientific World Journal
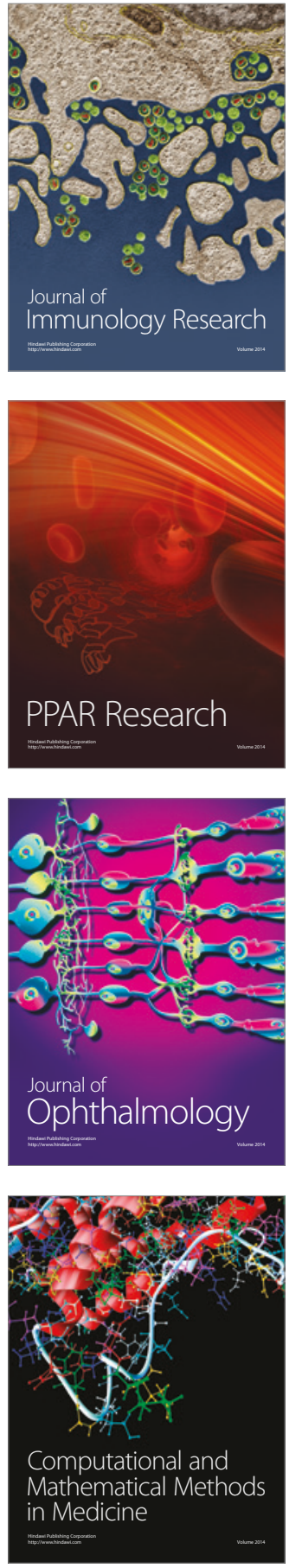

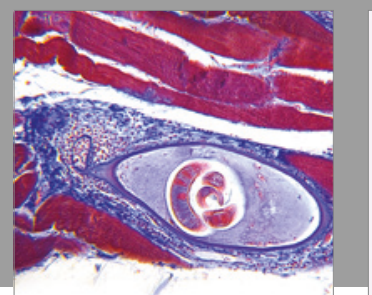

Gastroenterology Research and Practice
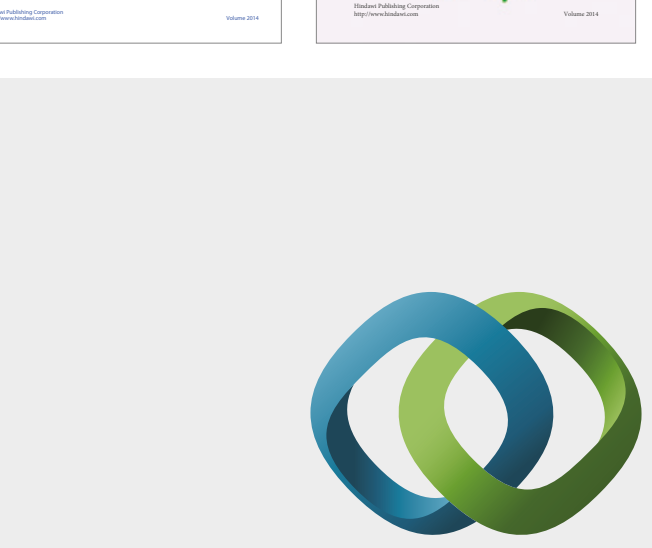

\section{Hindawi}

Submit your manuscripts at

https://www.hindawi.com
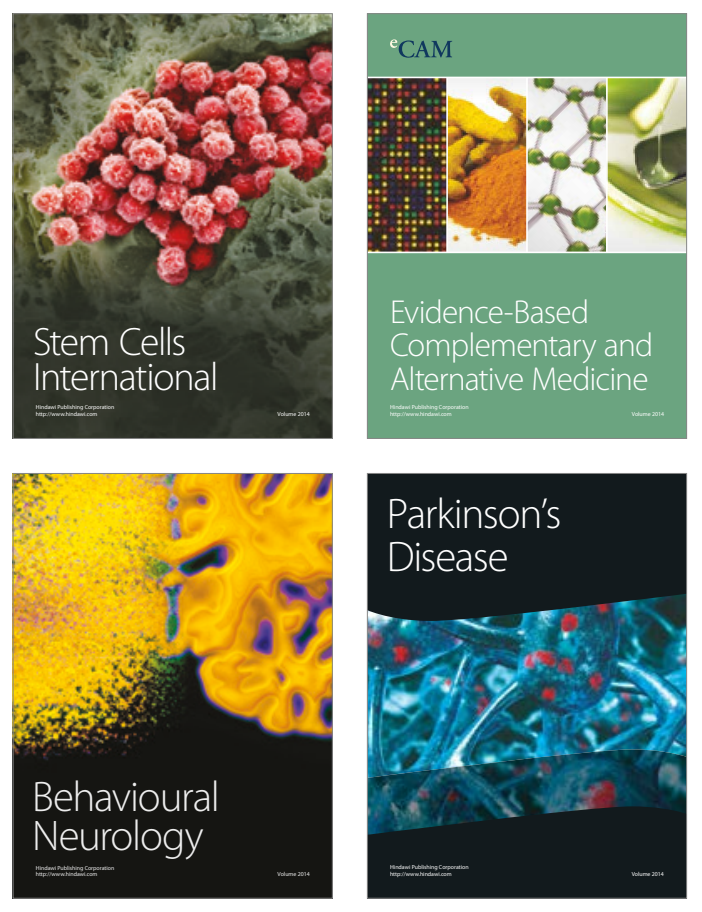
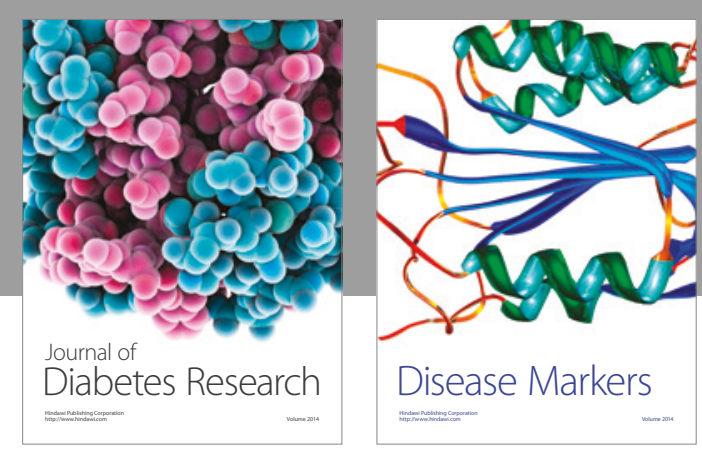

Disease Markers
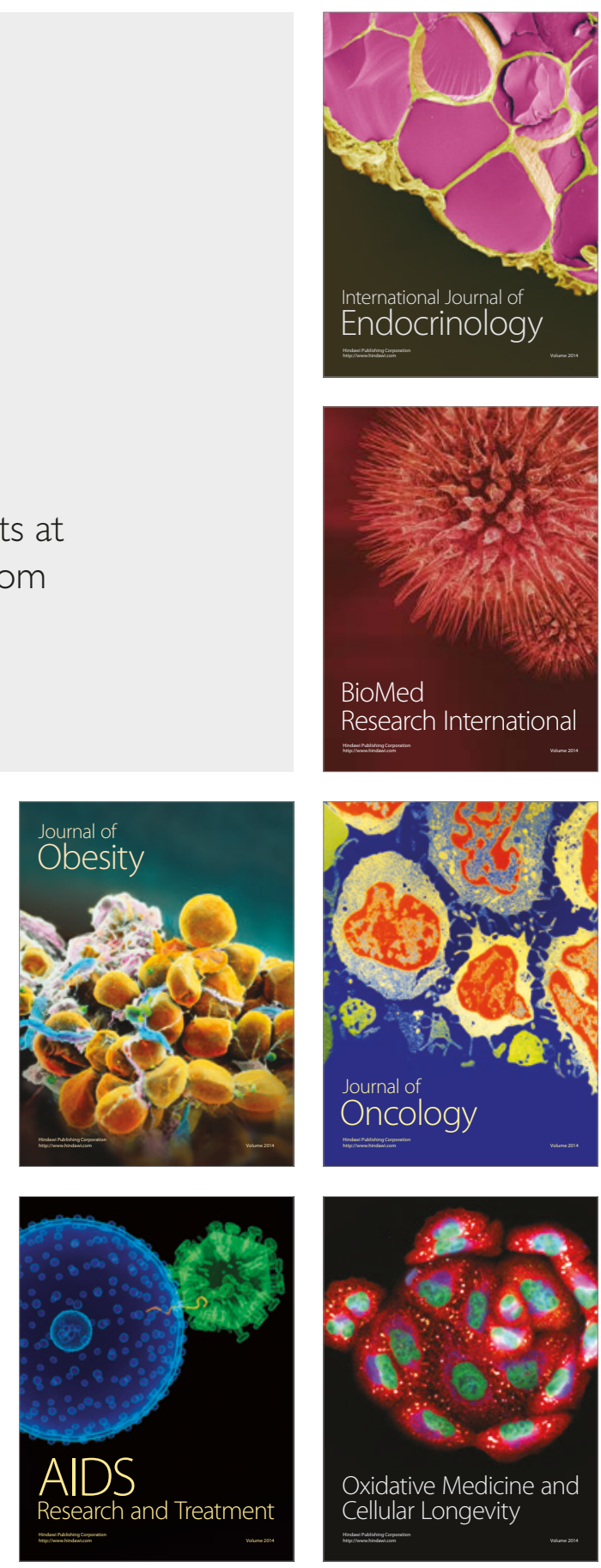